\title{
一类 Lotka-Volterra 系统正拟周期解的存在性
}

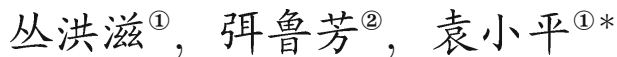

(1) 复旦大学数学科学学院, 上海 200433 ;

(2) 滨州学院数学与信息科学系, 滨州 256604

E-mail: xpyuan@fudan.edu.cn

收稿日期: 2008-11-25；接受日期: 2009-02-24; * 通信作者

国家自然科学基金 (批准号: 10725103)、111 引智计划和山东省教育厅科技计划（批准号: J07WH01) 资助项目

摘要 本文利用 KAM 迭代技巧证明了一类具有拟周期系数的 Lotka-Volterra 系统正拟周期解的存 在性.

关键词 拟周期解 Lotka-Volterra 系统 KAM 理论

MSC (2000) 主题分类 $37 \mathrm{~N} 25,92 \mathrm{D} 25,34 \mathrm{C} 27$

\section{1 引言}

考虑 Lotka-Volterra 系统

$$
\left\{\begin{array}{l}
\frac{d p}{d t}=\left(\lambda_{1}+\tilde{h}_{1}(t)\right) p+\left(-\lambda_{3}+\tilde{h}_{3}(t)\right) p q \\
\frac{d q}{d t}=\left(-\lambda_{2}+\tilde{h}_{2}(t)\right) q+\left(\lambda_{4}+\tilde{h}_{4}(t)\right) p q
\end{array}\right.
$$

其中 $\lambda_{i}(i=1,2,3,4)$ 是正常数, $\tilde{h}_{i}(t)(i=1,2,3,4)$ 是关于时间 $t$ 的具有相同周期的函数. LotkaVolterra 模型在生态系统中有很重要的应用, 它可以用来描述两个相互影响的生物种群的数量随时间 变化的情况. 很多学者运用各种各样的方法, 如拓扑度理论、不动点理论、Poincaré-Birkhoff 定理、变 分法等, 广泛深入地研究过这个模型的周期解的存在性、唯一性、稳定性以及其他相关的一些性质 (参 见文献 [1-6]). 上述文章考虑的都是周期 Lotka-Volterra 系统, 但是事实上, $\tilde{h}_{i}$ 的频率往往是不可公度 的, 或者说是拟周期的. 例如, $\tilde{h}_{1}, \ldots, \tilde{h}_{4}$ 的频率分别是 $\pi, 1, \sqrt{2}, \pi^{2}$. 在这种情形下, 通常希望系统存 在拟周期解而不是周期解. 当构建系统 (1) 的拟周期解时, 会出现一个所谓的小除数问题. 众所周知, $\mathrm{KAM}$ 理论是克服小除数问题的有力工具. 在本文中, 将应用 KAM 迭代技巧构造出系统 (1) 的正拟 周期解.

在给出定理之前, 先介绍一些概念和记号. 函数 $\mathcal{F}(\theta)=\mathcal{F}\left(\theta_{1}, \ldots, \theta_{n}\right)$ 关于每个变量以 $2 \pi$ 为周期, 若存在 $\omega=\left(\omega_{1}, \ldots, \omega_{n}\right)$, 使得 $F(t)=\mathcal{F}\left(\omega_{1} t, \ldots, \omega_{n} t\right) \in \mathbb{R}^{n}$, 则称 $F(t)$ 是拟周期的. 相应地, 称 $\omega$ 是 $F(t)$ 的频率, $\mathcal{F}(\theta)$ 是 $F(t)$ 的壳. 另外, 如果 $\mathcal{F}(\theta)$ 在复带状区域 $|\operatorname{Im} \theta| \leqslant \sigma$ 上解析, 且如果当 $\theta$ 为实 的时候函数 $\mathcal{F}(\theta)$ 也是实的, 则称 $F(t)$ 在宽度为 $\sigma$ 的带状区域内实解析. 为方便起见, 记

$$
\|F(t)\|_{\sigma}:=\|\mathcal{F}(\theta)\|_{\sigma}:=\sup _{|\operatorname{Im} \theta| \leqslant \sigma}|\mathcal{F}(\theta)| .
$$


定理 1.1 给定正常数 $\lambda_{i}(i=1,2,3,4), 0<\epsilon \ll 1$ 以及 $\mathbb{R}^{n}$ 中的一个具有正 Lebesgue 测度的紧 集 $\Pi$. 假设 $\tilde{h}_{i}(t)(i=1,2,3,4)$ 是以 $\omega=\left(\omega_{1}, \ldots, \omega_{n}\right) \in \Pi$ 为频率的拟周期函数, 且在宽度为 $\sigma>0$ 的 带状区域内实解析. 若 $\left\|\tilde{h}_{j}(t)\right\|_{\sigma} \leqslant \epsilon$, 则对大多数的 $\omega \in \Pi$ (在 Lebesgue 测度意义下), 系统 (1) 存在一 个以 $\omega$ 为频率的正拟周期解.

本文做如下安排: 在第 2 节中, 对系统 (1) 做一系列的坐标变换, 将方程变成便于应用 KAM 迭 代的形式; 在第 3 节中, 证明了一个迭代引理, 这是做 KAM 迭代的基础; 在第 4 节中, 根据迭代引理 完成了定理 1.1 的证明; 最后, 在附录中给出一些技术性的引理.

\section{2 坐标变换}

由 $\left\|\tilde{h}_{j}(t)\right\|_{\sigma} \leqslant \epsilon$, 我们可重新记 $\tilde{h}_{j}(t)=\epsilon h_{j}(t)$, 其中 $\left\|h_{j}(t)\right\|_{\sigma} \leqslant 1$. 假设频率 $\omega \in \Pi$ 满足 Diophantine 条件:

$$
|\langle k, \omega\rangle| \geqslant \gamma|k|^{-(n+1)}, \quad \forall 0 \neq k \in \mathbb{Z}^{n},
$$

否则挖掉那些不满足 Diophantine 条件的 $\omega$. 易证被挖掉的集合的测度小于或等于

$$
\sum_{k \in \mathbb{Z}^{n}}|k|^{-(n+1)} \gamma \leqslant C \gamma
$$

设 $\widehat{h}_{j}(k)$ 是 $h_{j}(t)$ 的壳的 $k$-Fourier 系数. 不失一般性, 可设 $\widehat{h}_{j}(0)=0$, 否则, 我们用 $\lambda_{i}+\widehat{h}_{i}(0)$ 替换 $\lambda_{i}$. 将 $h_{j}(t)$ 按照 Fourier 级数展开:

$$
h_{j}(t)=\sum_{k \in \mathbb{Z}^{r} \backslash\{0\}} \widehat{h}_{j}(k) e^{\sqrt{-1}\langle k, \omega\rangle t} .
$$

由于 $\omega$ 满足 Diophantine 条件, 且 $h_{j}(t)$ 在宽度为 $\sigma_{0}>0$ 的带状区域内实解析, 所以

$$
\int_{0}^{t} h_{j}(t) d t=\sum_{k \in \mathbb{Z}^{r} \backslash\{0\}} \frac{\widehat{h}_{j}(k)}{\sqrt{-1}\langle k, \omega\rangle}\left(e^{\sqrt{-1}\langle k, \omega\rangle t}-1\right)
$$

关于 $t$ 也是解析的拟周期函数. 做线性拟周期坐标变换

$$
p=e^{\int_{0}^{t} \epsilon h_{1}(s) d s} \tilde{p}, \quad q=e^{\int_{0}^{t} \epsilon h_{2}(s) d s} \tilde{q},
$$

方程组. (1) 变为

其中

$$
\left\{\begin{array}{l}
\frac{d \tilde{p}}{d t}=\lambda_{1} \tilde{p}+\left(-\lambda_{3}+\epsilon h_{5}(t)\right) \tilde{p} \tilde{q} \\
\frac{d \tilde{q}}{d t}=-\lambda_{2} \tilde{q}+\left(\lambda_{4}+\epsilon h_{6}(t)\right) \tilde{p} \tilde{q}
\end{array}\right.
$$

$$
\begin{aligned}
& h_{5}(t)=h_{3}+\epsilon^{-1}\left(-\lambda_{3}+\epsilon h_{3}\right)\left(e^{\int_{0}^{t} \epsilon h_{2}(t) d t}-1\right), \\
& h_{6}(t)=h_{4}+\epsilon^{-1}\left(-\lambda_{4}+\epsilon h_{4}\right)\left(e^{\int_{0}^{t} \epsilon h_{1}(t) d t}-1\right) .
\end{aligned}
$$

令

$$
\tilde{p}=\frac{\lambda_{2}}{\lambda_{4}} e^{\epsilon x_{1}}, \quad \tilde{q}=\frac{\lambda_{1}}{\lambda_{3}} e^{\epsilon x_{2}},
$$

198 
则方程组 (3) 变为

$$
\left\{\begin{array}{l}
\epsilon \frac{d x_{1}}{d t}=\lambda_{1}+\left(-\lambda_{1}+\frac{\lambda_{1}}{\lambda_{3}} \epsilon h_{5}(t)\right) e^{\epsilon x_{2}} \\
\epsilon \frac{d x_{2}}{d t}=-\lambda_{2}+\left(\lambda_{2}+\frac{\lambda_{2}}{\lambda_{4}} \epsilon h_{6}(t)\right) e^{\epsilon x_{1}}
\end{array}\right.
$$

利用 Taylor 公式, 有

$$
\left\{\begin{array}{l}
\frac{d x_{1}}{d t}=\left(-\lambda_{1}+\epsilon \xi_{1}(t)\right)\left(x_{2}+\int_{0}^{1} \int_{0}^{1} \epsilon^{2} s x_{2}^{2} e^{\epsilon s t x_{2}} d s d t\right)+\xi_{1}(t), \\
\frac{d x_{2}}{d t}=\left(\lambda_{2}+\epsilon \xi_{2}(t)\right)\left(x_{1}+\int_{0}^{1} \int_{0}^{1} \epsilon^{2} s x_{1}^{2} e^{\epsilon s t x_{1}} d s d t\right)+\xi_{2}(t),
\end{array}\right.
$$

其中 $\xi_{1}(t)=\frac{\lambda_{1}}{\lambda_{3}} h_{5}(t), \xi_{2}(t)=\frac{\lambda_{2}}{\lambda_{4}} h_{6}(t)$. 为方便起见, 重新记方程组 (6) 为

$$
\frac{d x}{d t}=(A+B(t)) x+\xi(t)+F(t, x),
$$

其中

$$
\begin{aligned}
& A=\left(\begin{array}{cc}
0 & -\lambda_{1} \\
\lambda_{2} & 0
\end{array}\right), B(t)=\left(\begin{array}{cc}
0 & \epsilon \xi_{1}(t) \\
\epsilon \xi_{2}(t) & 0
\end{array}\right), x=\left(\begin{array}{l}
x_{1} \\
x_{2}
\end{array}\right), \quad \xi(t)=\left(\begin{array}{l}
\xi_{1}(t) \\
\xi_{2}(t)
\end{array}\right), \\
& F(t, x)=\left(\begin{array}{c}
\left(-\lambda_{1}+\epsilon \xi_{1}(t)\right) \int_{0}^{1} \int_{0}^{1} \epsilon^{2} s x_{2}^{2} e^{\epsilon s t x_{2}} d s d t \\
\left(\lambda_{2}+\epsilon \xi_{2}(t)\right) \int_{0}^{1} \int_{0}^{1} \epsilon^{2} s x_{1}^{2} e^{\epsilon s t x_{1}} d s d t
\end{array}\right) .
\end{aligned}
$$

则我们可以直接得到

$$
\|F(t, x)\|_{\sigma_{0}} \leqslant C \epsilon, \quad\|F(t, x)\|_{\sigma_{0}}=O\left(\|x\|^{2}\right) .
$$

\section{3 迭代引理}

本节将给出一个 $\mathrm{KAM}$ 迭代引理, 为此引进一些迭代常数:

1. $\epsilon_{0}=\epsilon, \ldots, \epsilon_{l}=\epsilon^{(1+\rho)^{l}}, \ldots, l=0,1,2, \ldots, \rho=1 / 3$.

2. $\tau_{0}=0, \tau_{l}=\left(3^{-1}+\cdots+3^{-l}\right) / 2 \sum_{j=1}^{\infty} 3^{-j}$ (于是, $\tau_{l}<1 / 2$ 对所有 $l$ 成立).

3. $\sigma_{0}=\sigma, \ldots, \sigma_{l}=\left(1-\tau_{l}\right) \sigma_{0}, \ldots$ (于是, $\sigma_{l}>\sigma_{0} / 2$ 对所有 $l$ 成立).

4. $\beta_{0}=\beta_{0}, \ldots, \beta_{l}=\left(1-\tau_{l}\right) \beta_{0}, \ldots$ (于是, $\beta_{l}>\beta_{0} / 2$ 对所有 $l$ 成立).

5. $\iota_{l}=\sigma_{l}-\sigma_{l+1}$.

6. $\gamma_{0}=\gamma, \ldots, \gamma_{l}=\gamma / l^{2}, \ldots$

另外引入下列记号:

7. $\mathbb{T}^{n}=\mathbb{R}^{n} /(2 \pi \mathbb{Z})^{n}, \hat{\mathbb{T}}^{n}=\mathbb{C}^{n} /(2 \pi \mathbb{Z})^{n}$.

8. 对 $\sigma>0, \beta>0$, 令

$$
\begin{aligned}
& D(\sigma)=\left\{\theta \in \hat{\mathbb{T}}^{n}:|\operatorname{Im} \theta|=\max _{i}\left|\operatorname{Im} \theta_{i}\right|<\sigma\right\}, \\
& U(\sigma, \beta)=\left\{(\theta, x) \in \hat{\mathbb{T}}^{n} \times \mathbb{K}^{2}:|\operatorname{Im} \theta|<\sigma,|x|<\beta\right\},
\end{aligned}
$$


其中 $\mathbb{K}$ 表示 $\mathbb{C}$ 或 $\mathbb{R}$. 于是, 我们可以得到一族区域:

$$
D\left(\sigma_{0}\right) \supset D\left(\sigma_{1}\right) \supset \cdots \supset D\left(\sigma_{l}\right) \supset D\left(\sigma_{l+1}\right) \supset \cdots \supset D\left(\frac{\sigma_{0}}{2}\right)
$$

和

$$
U\left(\sigma_{0}, \beta_{0}\right) \supset U\left(\sigma_{1}, \beta_{1}\right) \supset \cdots \supset U\left(\sigma_{l}, \beta_{l}\right) \supset U\left(\sigma_{l+1}, \beta_{l+1}\right) \supset \cdots \supset U\left(\frac{\sigma_{0}}{2}, \frac{\beta_{0}}{2}\right) .
$$

9. $\Pi \in \mathbb{R}^{n}$ 是一正测参数集. 如果函数 $F: U(\sigma, \beta) \times \Pi \rightarrow \mathbb{K}$ 关于变量 $(\theta, x) \in U(\sigma, \beta)$ 解析, 关于 参数 $\omega \in \Pi$ 在 Whitney 意义下光滑, 且存在 $m \in \mathbb{Z}_{+}$, 使得

$$
\sup _{U(\sigma, \beta) \times \Pi}\left|\partial_{\omega_{j}}^{l} F(\theta, x, \omega)\right| \leqslant C_{m}|x|^{m},
$$

其中 $C_{m}$ 是依赖于 $m$ 和 $l$ 的常数, $l=0,1$, 则记

$$
F=O_{\sigma, \beta, \Pi}\left(x^{m}\right) .
$$

10. 给定 $\epsilon>0$, 如果函数 $F: D(\sigma) \times \Pi \rightarrow \mathbb{K}$ 关于变量 $\theta \in D(\sigma)$ 实解析, 关于参数 $\omega \in \Pi$ 在 Whitney 意义下光滑, 且满足

$$
\sup _{D(\sigma) \times \Pi}\left|\partial_{\omega_{j}}^{l} F(\theta, \omega)\right| \leqslant C \epsilon,
$$

则记

$$
F=O_{\sigma, \Pi}(\epsilon)
$$

11. 若存在常数 $C$, 使得 $\|F\|_{\sigma} \leqslant C\|G\|_{\sigma}$, 则记 $F \lessdot G$.

引理 3.1 设 $\Pi \subset \mathbb{R}^{n}$ 是一具有正 Lebesgue 测度的紧集, 记集合

$$
\begin{aligned}
& U(k):=\left\{\omega \in \Pi:\left|\sqrt{-1}\langle k, \omega\rangle-\mu_{1}\right| \leqslant \frac{\gamma}{|k|^{\tau}}, 0 \neq k \in \mathbb{Z}^{n}, \tau>n+1\right\}, \\
& V(k):=\left\{\omega \in \Pi:\left|\sqrt{-1}\langle k, \omega\rangle-\mu_{2}+\mu_{3}\right| \leqslant \frac{\gamma}{|k|^{\tau}}, 0 \neq k \in \mathbb{Z}^{n}, \tau>n+1\right\} .
\end{aligned}
$$

若 $\mu_{i}(i=1,2,3)$ 关于参数 $\omega \in \Pi$ 在 Whitney 意义下是 $C^{1}$ - 光滑的, 并且满足

$$
\sup _{\omega \in \Pi}\left|\partial_{\omega} \mu_{i}\right| \lessdot \frac{1}{8}
$$

则有

$$
\text { Meas } \tilde{\Pi} \leqslant C \gamma(\text { Meas } \Pi),
$$

其中 $\tilde{\Pi}=\bigcup_{k}(U(k) \cup V(k)), C$ 是一绝对常数.

证明 由条件 (11) 可知,

$$
\left|\partial_{\omega}\left(\langle k, \omega\rangle-\mu_{i}\right)\right|>1 / 2, \quad k \neq 0,
$$

所以

$$
\text { Meas }(U(k)) \leqslant \frac{C \gamma}{|k|^{\tau}}(\text { Meas } \Pi) \text {. }
$$

因此

$$
\text { Meas }\left(\bigcup_{k} U(k)\right) \leqslant C \gamma(\text { Meas } \Pi) \text {. }
$$


类似地, 可得

$$
\text { Meas }\left(\bigcup_{k} V(k)\right) \leqslant C \gamma(\text { Meas } \Pi) \text {. }
$$

从而结论成立.

引理 3.2 假设存在一族参数集合

$$
\mathbb{R}^{n} \supset \Pi_{0} \supset \Pi_{1} \supset \cdots \supset \Pi_{l} \supset \cdots \supset \Pi_{m}
$$

其 Lebesgue 测度满足:

$$
\text { Meas } \Pi_{0}>0, \quad \text { Meas } \Pi_{l} \geqslant\left(\text { Meas } \Pi_{l-1}\right)\left(1-C \gamma_{l-1}\right), \quad 1 \leqslant l \leqslant m,
$$

和一族微分方程 $(E q)_{l}(l=0,1,2 \ldots, m)$

$$
\frac{d x}{d t}=\left(A+B^{l}(\theta)\right) x, \quad \theta=\omega t,
$$

其中, $A$ 是一 $2 \times 2$ 常值实矩阵, 且 $A$ 特征值满足 $\left|\lambda_{1}-\lambda_{2}\right|>1, B^{l}(\theta)$ 关于变量 $\theta \in D\left(\sigma_{l}\right)$ 实解析, 关 于参数 $\omega \in \Pi_{l}$ 在 Whitney 意义下是 $C^{1}$ - 光滑的, 且有以下形式

$$
B^{l}(\theta, \omega)=\sum_{i=0}^{l} B_{i}(\theta, \omega),
$$

其中 $B_{i}(\theta, \omega)=O_{\sigma_{i}, \Pi_{i}}\left(\epsilon_{i}\right)$. 则存在 $\Pi_{m}$ 的子集 $\Pi_{m+1}$ 满足

$$
\text { Meas } \Pi_{m+1} \geqslant\left(\text { Meas } \Pi_{m}\right)\left(1-C \gamma_{m}\right),
$$

且对任意的 $\omega \in \Pi_{m+1}$ 存在一个线性变换 $x=P^{m}(\theta, \omega) y$, 将方程

$$
\dot{x}=\left(A+B^{m}(\theta, \omega)\right) x, \quad \theta=\omega t
$$

变成

$$
\dot{y}=\left(A^{m+1}+\tilde{B}_{m+1}(\theta, \omega)\right) y, \quad \theta=\omega t
$$

其中 $A^{m+1}=A+\sum_{i=0}^{m} O\left(\epsilon_{i}\right)$ 关于参数 $\omega \in \Pi_{m+1}$ 在 Whitney 意义下 $C^{1}$ - 光滑与 $\theta$ 无关,

$$
\tilde{B}_{m+1}(\theta, \omega)=O_{\sigma_{m+1}, \Pi_{m+1}}\left(\epsilon_{m+1}\right), \quad P^{m}(\theta, \omega)=\Pi_{i=0}^{m}\left(E+P_{i}(\theta, \omega)\right),
$$

$E$ 是个 $2 \times 2$ 单位矩阵, $P_{i}(\theta, \omega)=O_{\sigma_{i+1}, \Pi_{i+1}}\left(\gamma_{i}^{-1} \epsilon_{i} / \iota_{i}^{\tau+n}\right)$. 此外, 我们还可以得到

$$
\left\|P^{m}(\theta, \omega)\right\|_{\sigma_{m+1}, \Pi_{m+1}}, \quad\left\|\left(P^{m}(\theta, \omega)\right)^{-1}\right\|_{\sigma_{m+1}, \Pi_{m+1}} \leqslant 2 .
$$

证明 重新记方程 (12) 为

$$
\dot{x}=\left(A+B^{m-1}+B_{m}\right) x .
$$


由归纳假设, 存在一个线性变换 $x=P^{m-1} \tilde{y}$ 将方程

$$
\dot{x}=\left(A+B^{m-1}\right) x
$$

变成

$$
\dot{\tilde{y}}=\left(A^{m}+\breve{B}_{m}\right) \tilde{y}
$$

其中 $\breve{B}_{m}=O_{\sigma_{m}, \Pi_{m}}\left(\epsilon_{m}\right)$. 当 $m=0$ 时, 我们有 $B^{m-1}=0, P^{m-1}=E$.

记 $\tilde{B}_{m}=B_{m}+\breve{B}_{m}=O_{\sigma_{m}, \Pi_{m}}\left(\epsilon_{m}\right)$, 则存在变换 $y=\left(E+P_{m}\right) \tilde{y}$ 将

$$
\dot{\tilde{y}}=\left(A^{m}+\tilde{B}_{m}\right) \tilde{y}
$$

变成

$$
\frac{d y}{d t}=\left(E+P_{m}\right)^{-1}\left(A^{m}+\left(\tilde{B}_{m}-\dot{P}_{m}+A^{m} P_{m}\right)+\tilde{B}_{m} P_{m}\right) y .
$$

将 $\tilde{B}_{m}$ 按照 Fourier 级数展开 $\tilde{B}_{m}=\sum_{k \in \mathbb{Z}^{n}} \widehat{\tilde{B}_{m}}(k) e^{\sqrt{-1}\langle k, \omega\rangle t}$, 令

$$
A^{m+1}=A^{m}+\widehat{\tilde{B}_{m}}(0) .
$$

若 $P_{m}$ 满足方程

$$
\dot{P}_{m}=\tilde{B}_{m}+A^{m} P_{m}-P_{m} A^{m}-\widehat{\tilde{B}_{m}}(0)
$$

则方程 $(13)$ 变为

$$
d y / d t=A^{m+1} y+\left(E+P_{m}\right)^{-1} \tilde{B}_{m} P_{m} y
$$

由方程 (14) 和 $\tilde{B}_{m}=O_{\sigma_{m}, \Pi_{m}}\left(\epsilon_{m}\right)$, 我们可以得到

$$
A^{m+1}=A^{m}+O\left(\epsilon_{m}\right)=A+\sum_{i=0}^{m} O\left(\epsilon_{i}\right) .
$$

因为 $A$ 的特征值满足 $\left|\lambda_{1}-\lambda_{2}\right|>1$, 因此 $A^{m}$ 也满足 $\left|\lambda_{1}^{m}-\lambda_{2}^{m}\right|>1$. 从而 $A^{m}$ 可以对角化, 即存在 一个可逆矩阵 $M$, 使得 $M^{-1} A^{m} M=\Lambda$, 其中 $\Lambda=\operatorname{diag}\left(\lambda_{1}^{m}, \lambda_{2}^{m}\right)$. 根据附录中的引理 A.2, 我们有 $\|M\|,\left\|M^{-1}\right\| \lessdot 1$. 在方程 (15) 两端分别左乘 $M^{-1}$ 和右乘 $M$, 则方程 $(15)$ 变为

$$
\dot{P}=B+\Lambda P-P \Lambda-\widehat{B}(0)
$$

其中 $B=M^{-1} \tilde{B}_{m} M, P=M^{-1} P_{m} M$. 记

$$
B=\sum_{0 \neq k \in \mathbb{Z}^{n}} \widehat{B}(k) e^{\sqrt{-1}\langle k, \omega\rangle t}, \quad P=\sum_{0 \neq k \in \mathbb{Z}^{n}} \widehat{P}(k) e^{\sqrt{-1}\langle k, \omega\rangle t} .
$$

当 $k=0, i=j$ 时, 令

$$
P_{i i}(0)=0, \quad i=1,2
$$

否则, 有

$$
\widehat{P_{i j}}(k)=\frac{\widehat{B_{i j}}(k)}{\sqrt{-1}\langle k, \omega\rangle+\lambda_{i}^{m}-\lambda_{j}^{m}}, \quad i, j=1,2,
$$


其中 $P=\left(P_{i j}\right), B=\left(B_{i j}\right)$. 记集合

$$
V^{i j}(k):=\left\{\omega \in \Pi_{m}:\left|\sqrt{-1}\langle k, \omega\rangle+\lambda_{i}^{m}-\lambda_{j}^{m}\right| \leqslant \frac{\gamma_{m}}{|k|^{\tau}}, 0 \neq k \in \mathbb{Z}^{n}, \tau>n+1\right\}, \quad i, j=1,2 .
$$

令 $\Pi_{m+1}:=\Pi_{m} \backslash\left(\bigcup_{k i j} V^{i j}(k)\right)$. 则由引理 3.1 , 我们可以得到

$$
\text { Meas } \Pi_{m+1} \geqslant\left(\text { Meas } \Pi_{m}\right)\left(1-C \gamma_{m}\right) .
$$

对任意 $\omega \in \Pi_{m+1}$, 我们有

$$
\left|\sqrt{-1}\langle k, \omega\rangle+\lambda_{i}^{m}-\lambda_{j}^{m}\right|>\frac{\gamma_{m}}{|k|^{\tau}}
$$

根据 (18) 和 (20) 式, 我们有

$$
\left|\widehat{P_{i j}}(k)\right|<\gamma_{m}^{-1}|k|^{\tau}\left|\widehat{B_{i j}}(k)\right|
$$

从而得到

$$
\|\widehat{P}(k)\| \lessdot \gamma_{m}^{-1}|k|^{\tau}\|\widehat{B}(k)\| .
$$

根据 $\tilde{B}_{m}(\theta, \omega)=O_{\sigma_{m}, \Pi_{m}}\left(\epsilon_{m}\right), B=M^{-1} \tilde{B}_{m} M$ 和 $\|M\|,\left\|M^{-1}\right\| \lessdot 1$, 我们有 $\|B\| \lessdot \epsilon_{m}$, 且 $B$ 在区域 $D\left(\sigma_{m}\right)$ 上实解析. 从而

$$
\|\widehat{B}(k)\| \lessdot e^{-|k| \sigma_{m}} \epsilon_{m}
$$

由附录中的引理 A.1, 直接计算得

$$
\begin{aligned}
\sup _{D\left(\sigma_{m+1}\right)}\|P\| & \leqslant \sum_{k \in \mathbb{Z}^{n}}\|\widehat{P}(k)\| e^{|k| \sigma_{m+1}} \\
& \lessdot \sum_{k \in \mathbb{Z}^{n}} \gamma_{m}^{-1}|k|^{\tau}\|\widehat{B}(k)\| e^{|k| \sigma_{m+1}} \\
& \lessdot \epsilon_{m} \gamma_{m}^{-1}|k|^{\tau} e^{-|k|\left(\sigma_{m}-\sigma_{m+1}\right)} \\
& \lessdot \gamma_{m}^{-1} \epsilon_{m} / \iota_{m}^{\tau+n} .
\end{aligned}
$$

因此, $P$ 是方程 (17) 的唯一解且 $P=O_{\sigma_{m+1}, \Pi_{m+1}}\left(\gamma_{m}^{-1} \epsilon_{m} / \iota_{m}^{\tau+n}\right)$, 进而

$$
P_{m}=M P M^{-1}=O_{\sigma_{m+1}, \Pi_{m+1}}\left(\gamma_{m}^{-1} \epsilon_{m} / \iota_{m}^{\tau+n}\right)
$$

是方程 (15) 的唯一解. 令

$$
\tilde{B}_{m+1}=\left(E+P_{m}\right)^{-1} \tilde{B}_{m} P_{m}, \quad P^{m}=\left(E+P_{m}\right) \circ P^{m-1},
$$

则可以得到

$$
\tilde{B}_{m+1}=O_{\sigma_{m+1}, \Pi_{m+1}}\left(\gamma_{m}^{-1} \epsilon_{m}^{2} / \iota_{m}^{\tau+n}\right)=O_{\sigma_{m+1}, \Pi_{m+1}}\left(\epsilon_{m+1}\right)
$$

和

$$
\begin{aligned}
\left\|P^{m}(\theta, \omega)\right\|_{\sigma_{m+1}, \Pi_{m+1}} & \leqslant \Pi_{i=0}^{m}\left\|E+P^{i}(\theta, \omega)\right\|_{\sigma_{i+1}, \Pi_{i+1}} \\
& \leqslant \Pi_{i=0}^{m}\left(1+C \gamma_{i}^{-1} \epsilon_{i} / \iota_{i}^{\tau+n}\right) \leqslant 2 .
\end{aligned}
$$


另外容易得到

$$
\left\|\left(E+P_{i}(\theta, \omega)\right)^{-1}\right\| \leqslant 1+C \gamma_{i}^{-1} \epsilon_{i} / \iota_{i}^{\tau+n},
$$

因此我们有 $\left\|\left(P^{m}(\theta, \omega)\right)^{-1}\right\|_{\sigma_{m+1}, \Pi_{m+1}} \leqslant 2$.

最后, 我们将证明当 $\theta$ 是实的, 我们有 $P_{m}(\theta) \in \mathbb{R}$. 由归纳假设, 若 $\theta$ 是实的, 则 $A^{i}, P_{i}, B^{i}$ $(i \leqslant m-1)$ 也是实的. 从而可以得到 $A^{m}, \tilde{B}_{m}$ 也是实的. 由此, 易知 $\bar{P}_{m}(\theta)$ 是方程 (15) 的解, 其中 $\bar{P}_{m}(\theta)$ 是 $P_{m}(\theta)$ 的复共轭. 根据方程 (15) 解的唯一性, 我们可以得到 $\bar{P}_{m}(\theta)$ 和 $P_{m}(\theta)$ 在实轴上相等, 从而当 $\theta$ 是实的, $P_{m}(\theta)$ 也是实的. 到此我们完成了引理 3.2 的证明.

\section{4 定理 1.1 的证明}

为了证明定理 1.1, 我们先给出几个定义和引理:

定义 考虑平面系统 $\dot{x}=f(t, x, y), \dot{y}=g(t, x, y)$. 若存在定义在某个区间 $\mathcal{I}$ 的函数 $x=x(t), y=$ $y(t)$ 满足

$$
\sup _{t \in \mathcal{I}}\left|\frac{d x}{d t}-f(t, x(t), y(t))\right| \leqslant C \epsilon, \quad \sup _{t \in \mathcal{I}}\left|\frac{d y}{d t}-g(t, x(t), y(t))\right| \leqslant C \epsilon,
$$

则称 $x=x(t), y=y(t)$ 是这个系统的 $\epsilon$ - 近似解, 其中 $C$ 为常数.

引理 4.1 假设方程

$$
\frac{d x}{d t}=A(t) x+\xi(t)
$$

定义在区域 $D(\sigma)$ 上, 且 $A(t), \xi(t)$ 实解析. 任意给定一个解析, 有界且有有界逆的线性变换 $x=P(t) y$. 在这个变换下, 方程 $(21)$ 变为

$$
\dot{y}=P^{-1} A P y-P^{-1} \dot{P} y+P^{-1} \xi .
$$

并且, 如果 $y_{0}(t)$ 是方程 $(22)$ 的 $\epsilon$ - 近似解, 则 $P(t) y_{0}(t)$ 是方程 $(21)$ 的 $\epsilon$ - 近似解.

证明 对 $x=P y$ 关于 $t$ 求导, 则有

$$
\dot{x}=\dot{P} y+P \dot{y} .
$$

然后代入方程 (21), 我们就能得到方程 $(22)$.

若 $y_{0}(t)$ 是方程 $(22)$ 的 $\epsilon$ - 近似解, 则有

$$
\left|\dot{y}_{0}-P^{-1} A P y_{0}+P^{-1} \dot{P} y_{0}-P^{-1} \xi\right| \lessdot \epsilon .
$$

再因为 $\|P\|,\left\|P^{-1}\right\| \lessdot 1$, 直接计算得

$$
\begin{aligned}
\left|d\left(P y_{0}\right) / d t-A P y_{0}-\xi\right| & =\left|P \dot{y}_{0}+\dot{P} y_{0}-A P y_{0}-\xi\right| \\
& =\left|P\left(\dot{y}_{0}-P^{-1} A P y_{0}+P^{-1} \dot{P} y_{0}-P^{-1} \xi\right)\right| \\
& \leqslant\|P\| \cdot\left|\dot{y}_{0}-P^{-1} A P y_{0}+P^{-1} \dot{P} y_{0}-P^{-1} \xi\right| \\
& \lessdot \epsilon .
\end{aligned}
$$

因此 $P y_{0}(t)$ 是方程 $(21)$ 的 $\epsilon$ - 近似解.

引理 4.2 指定一个具有正 Lebesgue 测度的集合 $\Pi \in \mathbb{R}^{n}$ 以及一个正数 $\sigma \in \mathbb{R}$. 考虑微分方程

$$
\dot{x}=A(\omega) x+\xi(\theta, \omega), \quad \theta=\omega t,
$$


其中 $A(\omega)$ 是个 $2 \times 2$ 矩阵, 关于参数 $\omega \in \Pi$ 在 Whitney 意义下 $C^{1}$ - 光滑, 且两个特征值满足

$$
\left|\lambda^{1}(\omega)-\lambda^{2}(\omega)\right|>1, \quad \sup _{\omega \in \Pi}\left|\partial_{\omega} \lambda_{i}\right|<\frac{1}{8}, \quad i=1,2,
$$

并且函数 $\xi(\theta, \omega)=O_{\sigma, \Pi}(\epsilon)$ 是实解析、拟周期的. 则存在 $\Pi$ 的子集 $\Pi^{\prime}$ 满足

$$
\text { Meas } \Pi^{\prime} \geqslant(\text { Meas } \Pi)(1-C \gamma) \text {, }
$$

使得对任意 $\omega \in \Pi^{\prime}$, 方程 (23) 有一个实解析拟周期解 $x(t)=O_{\frac{3}{4} \sigma, \Pi^{\prime}}\left(\gamma^{-1} \epsilon\right)$.

证明 不失一般性, 我们假设 $A$ 是个对角矩阵, 且

$$
A=\left(\begin{array}{cc}
\lambda_{1} & 0 \\
0 & \lambda_{2}
\end{array}\right) .
$$

将 $x(\theta), \xi(\theta)$ 按照 Fourier 级数展开, 我们直接可以得到

$$
\widehat{x}_{i}(k)=\frac{\widehat{\xi}_{i}(k)}{\sqrt{-1}\langle k, \omega\rangle-\lambda_{i}}, \quad i=1,2,
$$

其中 $x=\left(x_{i}\right), \xi=\left(\xi_{i}\right), i=1,2$. 记集合

$$
U^{i}(k):=\left\{\omega \in \Pi:\left|\sqrt{-1}\langle k, \omega\rangle-\lambda_{i}\right| \leqslant \frac{\gamma}{|k|^{\tau}}, 0 \neq k \in \mathbb{Z}^{n}, \tau>n+1\right\}, \quad i=1,2,
$$

令 $\Pi^{\prime}:=\Pi \backslash\left(\bigcup_{k i} U^{i}(k)\right)$. 由引理 3.1 , 我们有

$$
\text { Meas } \Pi^{\prime} \geqslant(\text { Meas } \Pi)(1-C \gamma) \text {. }
$$

对任意 $\omega \in \Pi^{\prime}$, 我们有

$$
\left|\sqrt{-1}\langle k, \omega\rangle-\lambda_{i}\right|>\frac{\gamma}{|k|^{\tau}}
$$

根据方程 (25) 和 (27), 我们得到

$$
\left|\widehat{x_{i}}(k)\right|<\gamma^{-1}|k|^{\tau}\left|\widehat{\xi}_{i}(k)\right|
$$

从而

$$
\|\widehat{x}(k)\| \lessdot \gamma^{-1}|k|^{\tau}\|\widehat{\xi}(k)\| .
$$

因为有 $\xi(\theta)=O_{\sigma, \Pi}(\epsilon)$, 所以 $\|\widehat{\xi}(k)\| \lessdot \epsilon e^{-|k| \sigma}$ 成立. 由附录中的引理 A.1, 得

$$
\begin{aligned}
\sup _{D\left(\frac{3}{4} \sigma\right)}\|x\| & \leqslant \sum_{k \in \mathbb{Z}^{n}}\|\widehat{x}(k)\| e^{|k| \frac{3}{4} \sigma} \\
& \lessdot \sum_{k \in \mathbb{Z}^{n}} \gamma^{-1}|k|^{\tau}\|\widehat{\xi}(k)\| e^{|k| \frac{3}{4} \sigma} \\
& \lessdot \epsilon \gamma^{-1}|k|^{\tau} e^{-|k|\left(\sigma-\frac{3}{4} \sigma\right)} \\
& \lessdot \gamma^{-1} \epsilon .
\end{aligned}
$$

类似引理 3.2 的证明, 当 $\theta$ 是实的, 我们有 $x(\theta) \in \mathbb{R}$. 这样我们就完成了引理的证明. 
考虑方程 (7) 的线性化方程

$$
\frac{d x}{d t}=A x+\xi(t)
$$

根据引理 4.2 , 存在 $\Pi_{0}$ 的子集 $\Pi_{1}$ 满足

$$
\text { Meas } \Pi_{1} \geqslant\left(\text { Meas } \Pi_{0}\right)\left(1-C \gamma_{0}\right) \text {, }
$$

使得对任意的 $\omega \in \Pi_{1}$, 方程 (28) 有一个解 $x_{0}(t)=x^{0}(t)=O_{\sigma_{1}, \Pi_{1}}\left(\gamma_{0}^{-1} / \iota_{0}^{\tau+n}\right)$. 由方程 (8) 和 (10), 我 们可以得到 $x_{0}$ 是方程 (7) 的 $\epsilon$ - 近似解.

由数学归纳法, 我们假设存在一族参数集合

$$
\mathbb{R}^{n} \supset \Pi_{0} \supset \Pi_{1} \supset \cdots \supset \Pi_{l} \supset \cdots \supset \Pi_{m+1}
$$

满足

$$
\text { Meas } \Pi_{0}>0, \quad \text { Meas } \Pi_{l} \geqslant\left(\text { Meas } \Pi_{l-1}\right)\left(1-C \gamma_{l-1}\right), \quad 1 \leqslant l \leqslant m+1,
$$

使得对任意的 $\omega \in \Pi_{m+1}$, 方程 (7) 有一个实解析, 拟周期的 $\epsilon_{m^{-}}$近似解 $x^{m}=\sum_{i=0}^{m} x_{i}$, 且有 $x_{i}=$ $O_{\sigma_{i+1}, \Pi_{i+1}}\left(\gamma_{i}^{-1} \epsilon_{i-1} / \iota_{i}^{\tau+n}\right)$, 其中 $\epsilon_{-1}=1$.

将 $x=x^{m}+\tilde{x}$ 代入方程 (7), 有

$$
\begin{aligned}
\frac{d x^{m}}{d t}+\frac{d \tilde{x}}{d t}= & (A+B(t))\left(x^{m}+\tilde{x}\right)+\xi(t)+F\left(t, x^{m}+\tilde{x}\right) \\
= & (A+B(t)) x^{m}+\xi(t)+F\left(t, x^{m}\right)+(A+B(t)) \tilde{x}+\partial_{x} F\left(t, x^{m}\right) \tilde{x} \\
& +\int_{0}^{1} \int_{0}^{1} \partial_{x}^{2} F\left(t, \mu \nu \tilde{x}+x^{m}\right) \mu \tilde{x}^{2} d \mu d \nu .
\end{aligned}
$$

我们得到一个新的方程

$$
\frac{d \tilde{x}}{d t}=\left(A+B(t)+\partial_{x} F\left(t, x^{m}\right)\right) \tilde{x}+\xi^{m+1}(t)+\Upsilon^{m+1}(t, \tilde{x}),
$$

其中

$$
\begin{aligned}
& \xi^{m+1}(t)=\frac{d x^{m}}{d t}-\left((A+B(t)) x^{m}+\xi(t)+F\left(t, x^{m}\right)\right), \\
& \Upsilon^{m+1}(t, \tilde{x})=\int_{0}^{1} \int_{0}^{1} \partial_{x}^{2} F\left(t, \mu \nu \tilde{x}+x^{m}\right) \mu \tilde{x}^{2} d \mu d \nu=O_{\sigma_{m+1}, \Pi_{m+1}}\left(\tilde{x}^{2}\right) .
\end{aligned}
$$

因为 $x^{m}(t)$ 是方程 (7) 的 $\epsilon_{m}$ - 近似解, 由定义有

$$
\xi^{m+1}(t)=O_{\sigma_{m+1}, \Pi_{m+1}}\left(\epsilon_{m}\right) .
$$

如果我们能找到线性化方程

$$
\frac{d \tilde{x}}{d t}=\left(A+B(t)+\partial_{x} F\left(t, x^{m}\right)\right) \tilde{x}+\xi^{m+1}(t) .
$$

的形式为 $x_{m+1}=O_{\sigma_{m+2}, \Pi_{m+2}}\left(\gamma_{m+1}^{-1} \epsilon_{m} / \iota_{m+1}^{\tau+n}\right)$ 的 $\epsilon_{m+1}$ - 近似解. 由 (30), 我们有

$$
\Upsilon^{m+1}\left(t, x_{m+1}\right)=O_{\sigma_{m+2}, \Pi_{m+2}}\left(\left(\gamma_{m+1}^{-1} \epsilon_{m} / \iota_{m+1}^{\tau+n}\right)^{2}\right)=O_{\sigma_{m+2}, \Pi_{m+2}}\left(\epsilon_{m+1}\right) .
$$


从而 $x_{m+1}$ 也是方程 (29) 的 $\epsilon_{m+1}$ - 近似解. 则 $x^{m+1}=x^{m}+x_{m+1}$ 是方程 (7) 的 $\epsilon_{m+1}$ - 近似解. 为此, 我们先考虑线性项 $\partial_{x} F\left(t, x^{m}\right)$. 利用 Taylor 公式, 对所有 $1 \leqslant l \leqslant m$, 有

$$
\partial_{x} F\left(t, x^{l}\right)=\partial_{x} F\left(t, x^{l-1}\right)+\int_{0}^{1} \partial_{x}^{2} F\left(t, s x_{l}+x^{l-1}\right) x_{l} d s,
$$

和

$$
\int_{0}^{1} \partial_{x}^{2} F\left(t, s x_{l}+x^{l-1}\right) x_{l} d s=O\left(x_{l}\right)=O_{\sigma_{l+1}, \Pi_{l+1}}\left(\gamma_{l}^{-1} \epsilon_{l-1} / \iota_{l}^{\tau+n}\right) .
$$

因此, 对 $m$ 进行数学归纳, 从而有 $B(t)+\partial_{x} F\left(t, x^{m}(t)\right)=\sum_{i=0}^{m} B_{i}(t)$, 其中 $B_{i}$ 满足引理 3.2 中的假 设. 从而根据引理 3.2 , 存在 $\Pi_{m+1}$ 的一个子集 $\Pi_{m+1}^{\prime}$, 使得对任意的 $\omega \in \Pi_{m+1}^{\prime}$, 存在一个线性坐标变 换 $x=P^{m}(\theta, \omega) y$, 将方程

$$
\frac{d x}{d t}=\left(A+B(t)+\partial_{x} F\left(t, x^{m}\right)\right) x
$$

变为

$$
\dot{y}=\left(A^{m+1}+\tilde{B}_{m+1}(\theta, \omega)\right) y
$$

其中

$$
\begin{aligned}
& A^{m+1}(\omega)=A+\sum_{i=1}^{m} O_{\Pi_{i}}\left(\epsilon_{i}\right), \quad \tilde{B}_{m+1}(\theta, \omega)=O_{\sigma_{m+1}^{\prime}, \Pi_{m+1}^{\prime}}\left(\epsilon_{m+1}\right), \\
& \left\|P^{m}(\theta, \omega)\right\|_{\sigma_{m+1}^{\prime}, \Pi_{m+1}^{\prime}},\left\|\left(P^{m}(\theta, \omega)\right)^{-1}\right\|_{\sigma_{m+1}^{\prime}, \Pi_{m+1}^{\prime}} \leqslant 2 .
\end{aligned}
$$

再根据引理 4.2 , 存在 $\Pi_{m+1}^{\prime}$ 的子集 $\Pi_{m+2}$, 使得对任意的 $\omega \in \Pi_{m+2}$, 方程

$$
\dot{y}=A^{m+1} y+\left(P^{m}\right)^{-1} \xi^{m+1}
$$

有一个实解析拟周期解 $y(t)=O_{\sigma_{m+2}, \Pi_{m+2}}\left(\gamma_{m+1}^{-1} \epsilon_{m} / \iota_{m+1}^{\tau+n}\right)$, 且 $\Pi_{m+2}$ 满足

$$
\text { Meas } \Pi_{m+2} \geqslant\left(\text { Meas } \Pi_{m+1}^{\prime}\right)\left(1-C \gamma_{m+1}\right) \text {. }
$$

由引理 4.1 和方程 (32), 我们知道 $x_{m+1}=P^{m} y=O_{\sigma_{m+2}, \Pi_{m+2}}\left(\gamma_{m+1}^{-1} \epsilon_{m} / \iota_{m+1}^{\tau+n}\right)$ 是方程 (31) 的 $\epsilon_{m+1^{-}}$ 近似解. 因此, $x^{m+1}=x^{m}+x_{m+1}$ 也是方程 (7) 的 $\epsilon_{m+1}$-近似解. 要注意的是 $x_{i}(t)=x(t, \omega)$ 以及 $P_{i}=P_{i}(t, \omega)$ 关于 $t \in \mathbb{R}$ 是解析的、关于 $\omega \in \Pi_{i+1}$ 是在 Whitney 意义下 $C^{1}$ - 光滑的.

最后令

$$
\Pi_{\infty}=\bigcap_{i=0}^{\infty} \Pi_{i}, \quad x^{\infty}(t)=\lim _{m \rightarrow \infty} x^{m}(t, \omega)=\sum_{i=0}^{\infty} x_{i}(t, \omega),
$$

则当 $\omega \in \Pi_{\infty}$ 时, $x^{\infty}(t)=x^{\infty}(t, \omega)$ 是方程 (7) 的实解析拟周期解, 且有以下估计

$$
\begin{aligned}
\text { Meas } \Pi_{\infty} & \geqslant(\text { Meas } \Pi) \Pi_{i=0}^{\infty}\left(1-C \gamma_{i}\right) \\
& >(\text { Meas } \Pi)\left(1-\sum_{i=0}^{\infty} C \gamma_{i}\right) \\
& >(\text { Meas } \Pi)\left(1-C \gamma_{0}\right)
\end{aligned}
$$

和

$$
\left\|x^{\infty}\right\|_{\sigma_{0} / 2, \Pi_{\infty}} \lessdot C \gamma_{0}^{-1}\left(1+\sum_{i=0}^{\infty} \gamma_{i}^{-1} \epsilon_{i-1} / \iota_{i}^{\tau+n}\right) \lessdot \gamma_{0}^{-1} .
$$


记 $x^{\infty}(t)=\left(x_{1}^{\infty}(t), x_{2}^{\infty}(t)\right)$. 由 $(2)$ 和 $(4)$ 得,

$$
\left(\begin{array}{c}
p(t) \\
q(t)
\end{array}\right)=\left(\begin{array}{c}
e^{\int_{0}^{t} \epsilon h_{1}(s) d s} \tilde{p} \\
e^{\int_{0}^{t} \epsilon h_{2}(s) d s} \tilde{q}
\end{array}\right)=\left(\begin{array}{c}
\frac{\lambda_{2}}{\lambda_{4}} e^{\int_{0}^{t} \epsilon h_{1}(s) d s} \cdot e^{x_{1}^{\infty}(t)} \\
\frac{\lambda_{1}}{\lambda_{3}} e^{\int_{0}^{t} \epsilon h_{2}(s) d s} \cdot e^{x_{2}^{\infty}(t)}
\end{array}\right)
$$

是系统 (1) 的正拟周期解. 从而我们完成了定理 1.1 的证明.

致谢 作者感谢审稿人的宝贵建议。

\section{参考文献}

1 Ahmad S. On almost periodic solutions of the completing species problems. J Amer Math Soc, 1998, 102: 855-861

2 Alvarez C, Lazer A C. An application of topological degree to the periodic competing species problem. J Austral Math Soc Ser B, 1986, 28: 202-219

3 Bogoljubov N N, Mitropoliskii J A, Samoilenko A M. Methods of Accelerated Convergence in Nonlinear Mechanics. New York: Springer-Verlag, 1976

4 Ding T, Zanolin F. Periodic solutions and subharmonic solutions for a class of planar systems of Lotka-Volterra type. In: Lakshmikantham V, ed. Proceedings for the First World Congress of Nonlinear Analysts. Florid: Tampa, 1992, 19-26

5 Hausrath A R, Manásevich R F. Periodic solutions of a periodically perturbed Lotka-Volterra equation using the Poincaré-Birkhoff theorem. J Math Anal Appl, 1991, 157: 1-9

6 Liu L, Lu Z, Wang D. The structure of LaSalle's invariant set of Lotka-Volterra systems. Sci China Ser A, 1991, 34: 783-790

\section{附录}

引理 $\mathbf{A . 1}$ 对 $\delta>0, \nu>0$, 下列不等式成立:

$$
\sum_{k \in \mathbb{Z}^{n}} e^{-2|k| \delta}|k|^{\nu} \leqslant\left(\frac{\nu}{e}\right)^{\nu} \frac{1}{\delta^{\nu+n}}(1+e)^{n} .
$$

证明 引理的证明可参见文献 [3].

引理 $\mathbf{A . 2}$ 设矩阵 $T(\omega)=T_{0}+B(\omega)$, 其中

$$
T_{0}=\left(\begin{array}{cc}
0 & -\lambda_{1} \\
\lambda_{2} & 0
\end{array}\right), \quad B(\omega)=\left(\begin{array}{cc}
b_{11}(\omega) & b_{12}(\omega) \\
b_{21}(\omega) & b_{22}(\omega)
\end{array}\right),
$$

且 $\lambda_{i}(i=1,2)$ 是满足 $\lambda_{i}>c>0$ 的常数. 若 $B$ 关于参数 $\omega$ 光滑, 且 $\left\|\partial_{\omega}^{l} B(\omega)\right\| \leqslant \epsilon, l=0,1$. 记 $\mu_{i}[T](i=1,2)$ 是 $T(\omega)$ 的特征值, 则有 $\left|\partial_{\omega} \mu_{i}[T]\right| \lessdot \epsilon$, 且存在满足条件 $\|M(\omega)\|,\left\|M(\omega)^{-1}\right\| \lessdot 1$ 的 $M(\omega)$, 使得 $M(\omega)^{-1} T(\omega) M(\omega)$ 是 对角矩阵.

证明 首先, 直接计算可得

$$
\mu_{i}[T]=\frac{1}{2}\left\{b_{11}+b_{22} \pm \sqrt{\left(b_{11}+b_{22}\right)^{2}-4\left[b_{11} b_{22}-\left(b_{12}-\lambda_{1}\right)\left(b_{21}+\lambda_{2}\right)\right]}\right\}
$$

易证 $\left|\partial_{\omega} \mu_{i}[T]\right| \lessdot \epsilon$. 设 $X_{i}=\left(\begin{array}{c}x_{1}^{i} \\ x_{2}^{i}\end{array}\right)$ 是 $T(\omega)$ 对应于特征值 $\mu_{i}(T)$ 的特征向量. 取 $x_{1}^{i}=1$, 则 $x_{2}^{i}=\left(\mu_{i}-b_{11}\right) /\left(b_{12}-\lambda_{1}\right)$. 令

$$
M=\left(X_{1}, X_{2}\right)=\left(\begin{array}{cc}
1 & 1 \\
\left(\mu_{1}-b_{11}\right) /\left(b_{12}-\lambda_{1}\right) & \left(\mu_{2}-b_{11}\right) /\left(b_{12}-\lambda_{1}\right)
\end{array}\right),
$$

直接计算可得 $C_{1} \leqslant\|M\| \leqslant C_{2},\left\|\partial_{\omega} M\right\| \lessdot \epsilon$, 所以 $\|M(\omega)\|,\left\|M(\omega)^{-1}\right\| \lessdot 1$. 